\title{
Study on Coalmine Safety Behavior Control System Model and its Manager Behavior Choice
}

\author{
Qingren Cao1, Xiaobing Yang ${ }^{2}$ \\ ${ }^{1}$ School of Management, China University of Mining and Technology, Xuzhou, China; ${ }^{2}$ School of Art, Xuzhou Institute of Technol- \\ ogy, Xuzhou, China. \\ Email: caoqr-cumt@163.com,xbyang_711@yahoo.com.cn
}

Received May $17^{\text {th }}, 2009$; revised December $20^{\text {th }}$, 2009; accepted January $15^{\text {th }}, 2010$.

\begin{abstract}
To control coal miner's unsafe behavior, miner and manager behaviors were analyzed, and a behavior control system model was constructed. The necessity conditions of optimal management behavior of coalmine manager in both complete information and incomplete information condition were studied. The result established that, only if the estimated value of unsafe behavior by manager was the same as the estimated value of unsafe behavior by miner, namely both miner's behavior control and manager own behavior control were optimal, the optimal behavior control of coalmine manager were achieved. Because some factors influenced manager's behavior unavoidably, it is very difficult for coalmine manager to achieve his optimal behavior choice. It is the underlying reason for many managerial defects, even errors inherent in the coalmine safety management.
\end{abstract}

Keywords: Coalmine Manager, Behavior Control System, Optimization Model, Unsafe Behavior, Manager Behavior Choice

\section{Introduction}

Recently, major casualty accidents took place repeatedly in China has been paid attention all around the world. By investigating these accidents carefully, it was found that many managers supervised coal miner only by their experiences, and there were few scientific theories supporting their management behaviors. These behaviors are no doubt harmful to the coalmine safety management, but this is very common in China, so it is inevitable there are considerable managerial defects and errors existed in coalmine safety management. It was for this underlying reason that has caused casualty accidents took place repeatedly in China.

As we all know, the characteristic of coal production determines there are full of potentially dangerous in coal production process. In order to prevent possible accidents or reduce their harmful degree to an acceptable level, it is needed to remove the factors that might conduce to serious injury or loss of life by implementing various safety management measures, such as managing the essential factors that influence miner to do unsafe behavior. However, as one composition of coalmine safety management, behavior management is more important to physical management, because miner's behavior is a more active ingredient in the coal production process, and the miner's unsafe behavior is the primary and direct factors leading to coalmine accidents. In addition, there are always behavior mistakes underlying unsafe physical status. So the unsafe behavior management is always a more important method to prevent accidents in coalmine safety management [1].

In the coal production process, miner's behavior and manager's behavior are very different on the view of management. Although miner's unsafe behavior is the direct contributing factors to accidents, the miner's behavior is a management objective of the manager's behavior. So studying manager's behavior and its characteristics is even more important.

For a coalmine manager, there are a lot of available management measures, but different management measures have different effects. If a manager could not properly make use of his limited time, energy and other available resources, his management measures are difficult to achieve some certain or better results [2]. So the coalmine manager will be facing a theoretic problem how to make the right choice of different management measures scientifically.

In fact, many scholars have studied this kind of behavior choice problem for ages. Mintzberg may be the pioneer to study this kind of question. He divided the manager behavior into several work roles, and studied the relationship of time spent on these different roles and the work results [3]. 
Allan had surveyed 1476 New York City government managers for their more important jobs and time spent on them. He discovered that there are 57 core jobs needed to be accomplished in different positions or different departments. Along with the position moving up, the duty becomes extended accordingly, so the middle managers and the senior managers have 80 and 87 core jobs to be done respectively [4]. Yuk also studied this issue by similar method, but he divided the manager work jobs into 22 items [5].

Similar to these scholars, Schaechtel disclosed that safety management is only one job for a manager in many organizations, so how to force a manager pay more attention to the safety management work all the time is very important [6]. He deemed that it needed to construct a safety management system framework to measure or evaluate manager's safety performance. This framework should meet the requirement that the safety standard is delivered to every employee distinctly, the duty to implement standard is understood and accept, there are documents to record the implemented standard, and there are effective internal control mechanisms. He also brought forward a method to construct this kind of safety management system.

But Jens deemed that a risk management system consists of different members, such as lawmakers, supervisors, planners, and manipulators [7]. In the past, the system problems were studied in different areas respectively. To construct a control system model needed to generalize or sum up all these systems or their specific dangers. In effect, to construct a risk management system model, we must regard the risk management as a control problem and study them crossways. Only in that way can we consider all the relevant problems.

All these studies show that manager has many jobs to do, and he needed to make choice rightly because different behavior choice leads to different management effects. Unlike these studies, a behavior control system model was constructed by summarizing the influence factors of coal miner and manager from the point of behavior control mechanism in this paper [8]. How to make the optimal control behavior choice for coalmine manager was analyzed theoretically, the conditions of making the optimal control behavior choice were induced, and the characteristics of optimal control behavior were summed up. This is the theoretical basis for empirical research of coalmine manager's control behavior.

\section{Coal Miner Behavior System}

For a long time, many scholars have studied the factors that influence people to make unsafe behavior choice and behavior system. These studies help us to understand all kinds of human behaviors, and provide us with precondition of engaging in the safety management study nowadays.
Specific to the miner behavior, Jason has studied many variables to influence safety management measures put into practice, such as the miner's sensation to effectiveness of behavior safety training, the miner's trust to the ability of manager, the manager's responsibility to performance evaluation, the miner's relevant education experience, the manager's work time in the organization, etc. [9].

After detailing investigation, Michael discovered that several factors influenced the employee's sensation, such as the manager's duty [10]. By making use of these factors carefully, the manager could reduce the accidents to the lowest level. In the meantime, Geller studied the sensation, perception, pressure, suffering and complexity of human being, analyzed the behavior safety and its training on individual, and put forward four behavior intervention avenues in accordance with three kinds of behavior manner [11].

All these studies could help us to understand the miner behavior very well. But after all, the coal production task is fulfilled by the miner's actual production behavior. During his work, the miner's behavior is influenced or stimulated by many extrinsic factors. These factors may make the miner's mental state transferring continually by his perception. Furthermore they may impact the estimated value of behavior factors, which decide he is to adopt safe or unsafe behavior.

There are a lot of extrinsic factors influencing the coal miner behavior. These factors may be summed up five aspects, i.e. work circumstance, tasks and behavior criterion, incentive, rewards and punishment behavior, education and training, and group and organization behavior [12-14]. They also can be regarded as the input variables of coal miner behavior system. The first aspect indicates work objective and work circumstance of coal miner, but manager can change it by work design. The latter four aspects indicate the control measures available to the manager.

Coal miner behavior may be described as behavioral state and state transformation. In the coal production process, many factors stimulated miner behavior continuously, inspired different behavior motivation, and made the choice of different behaviors. It can be regarded as transformation from one state to another, meanwhile completing the coalmine production task.

The inner factors are fundamental to coal miner behavior. They may be summed up five aspects, i.e. miner's mental state, knowledge state, physical state, organizational loyalty and work effort. All of these factors together may be described as miner's safety consciousness and work capability that can be used as the miner's state variables [15]. The output of miner behavior system is the safe or unsafe behavior according to their estimated values. 
Suppose $x_{1 t}, x_{2 t}$ respectively denotes miner's safety consciousness and work capability in time $t(t=0,1,2 \ldots T)$, and $u_{1 t}, u_{2 t}, u_{3 t}, u_{4 t}, u_{5 t}$ respectively denotes task and behavior criterion provided by manager, manager's rewards and punishment behavior, education and training giving by manager, group or organization behavior shaped by manager, and working circumstance designed by manager. Then the miner's behavior state can be described as

$$
\boldsymbol{x}(t+1)=\boldsymbol{f}(\boldsymbol{x}(t), \boldsymbol{u}(t), t)
$$

There, $\boldsymbol{x}(t)=\left(x_{1 t}, x_{2 t}\right)^{\mathrm{T}}, \boldsymbol{u}(t)=\left(u_{1 t}, u_{2 t}, u_{3 t}, u_{4 t}, u_{5 t}\right)^{\mathrm{T}}, \boldsymbol{f}$ is a vector function.

The input variables and state variables of miner behavior system could influence the estimated value of unsafe behavior in the production process. Firstly, miner's safety consciousness could influence his selective perception, including selective perception of influencing factors of unsafe behavior. Work capability also influences his choice of unsafe behavior. Secondly, some factors in work circumstance could influence the estimated value of unsafe behavior, because miner's selective perception of work circumstance factors may strengthen or weaken his judgment of unsafe behavior cost, and the manager's rewards and punishment behavior could influence the miner's value judgment of unsafe behavior. Thirdly, task and behavior criterion, rewards and punishment behavior, education and training, and group or organization behaviors could influence the miner's value judgment of unsafe behavior by his behavior state variables.

Take the miner's estimated value of unsafe behavior as the function of its influencing factors in time $t(t=0$, $1,2 \ldots T)$, thus

$$
w(t)=g(\boldsymbol{x}(t), \boldsymbol{u}(t), t)
$$

The above (1) and (2) make for the description of coal miner behavior system.

\section{Coalmine Manager Behavior System}

Coalmine manager behavior is different from coal miner behavior, because coalmine manager accomplishes his task by influencing or controlling miner behavior using management knowledge and skills. In the management process, manager needs to keep access to a variety of information repeatedly, such as the work circumstance, miner behavior. Based on this information, the manager makes his own behavior choice to control the miner behavior. Manager behavior choice is also influenced by the incentive contract and the coalmine production task.

In order to maintain consistent with the extrinsic influencing factors of coalmine manager, the input of manager behavior system may be summarized as the following aspects, i.e. manager's cognition of work circumstance, manager's cognition of miner unsafe behavior, coalmine production task of manager, and manager's incentive contract [16]. The former two aspects are related to the coal production process, so they can be regarded as the information that the manager needed to complete his task of safety management, and belong to the controllable factors. The latter two aspects are not related to the coal production process, and are not related to completing the task of safety management, belonging to the uncontrollable factors.

The inner factors are fundamental to the coalmine manager. They may be summed up five aspects, i.e. manager's mental state, knowledge state, physical state, organizational loyalty and work effort. All these factors integrated may be described as manager's work initiative and work capability that can be used as the state variables of coalmine manager.

In order to maintain consistent with miner's unsafe behavior control in coal production process, the output of manager behavior system should be constituted by safety management measures that may be carried out by the manager, including the behavior choice of safety management and the work effort in that choice. The manager behavior consists of designing and providing production task and behavior criterion, carrying up rewards and punishment, bringing into effect of education and training, and creating group atmosphere and organizational climate.

Suppose $y_{1 t}, y_{2 t}$ respectively denotes the manager's work initiative and work capability in time $t \quad t=0$, $1,2 \ldots T)$, and $v_{1 t}, v_{2 t}, v_{3 t}, v_{4 t}$ respectively denotes manager's production task, manager's incentive contract, manager's cognition of work circumstance, manager's cognition of miner unsafe behavior. Then the manager's behavior state may be described as

$$
\boldsymbol{y}(t+1)=\boldsymbol{h}(\boldsymbol{y}(t), \boldsymbol{v}(t), t)
$$

There, $\boldsymbol{y}(t)=\left(y_{1 t}, y_{2 t}\right)^{\mathrm{T}}, \boldsymbol{v}(t)=\left(v_{1 t}, v_{2 t}, v_{3 t}, v_{4 t}\right)^{\mathrm{T}}, \boldsymbol{h}$ is a vector function.

The output of manager behavior system is mainly the safety management measures available to the manager, including designing and providing production task and behavior criterion, carrying up rewards and punishment, bringing into effect of education and training, and creating group atmosphere and organizational climate. Consistent with the miner behavior system, they are denoted as $u_{1}, u_{2 t}$, $u_{3}, u_{4 t}$ respectively. They are all extrinsic influencing factors for the miner and are influenced by the manager's behavior state variable and input variable. Thus

$$
\boldsymbol{u}^{\prime}(t)=\boldsymbol{h}^{\prime}(\boldsymbol{y}(t), \boldsymbol{v}(t), t)
$$

here, $\boldsymbol{u}^{\prime}(t)=\left(u_{1 t}, u_{2 t}, u_{3 t}, u_{4 t},\right)^{\mathrm{T}}, \boldsymbol{h}$ ' is a vector function.

The above (3), and (4) make for the description of coalmine manager behavior system.

\section{Safety Behavior Control System Model}

In fact, miner behavior system interacts with manager behavior system in the coal production process. The 
miner fulfills the coal production task assigned by the manager, in the same time making choice of safe or unsafe behavior based on his perception and judgment. The manager implements his management actions based on the work circumstance, work objective, and miner behavior, influencing or changing the miner's work circumstance and unsafe behavior.

The safety management measures available to the manager consist of designing and providing production task and behavior criterion, carrying up rewards and punishment, bringing into effect of education and training, and creating group atmosphere and organizational climate. They are all extrinsic factors that may influence the miner's behavior. Thus, directed by the coal production task, under the action of safety management measures available to the manager, miner behavior system and manager behavior system make up of an integrating safety behavior control system. The system model is described as in Figure 1.

There are two inputs in the coalmine safety behavior control system. One comes from the manager, including manager's production task, manager's incentive contract, manager's cognition of work circumstance, and manager's cognition of miner unsafe behavior. Although manager's cognition of miner unsafe behavior is an input of manager behavior system, it is not an input of the coalmine safety behavior control system. Another comes from the miner, which include the stimulation of work circumstance. Others are not the input of the coalmine safety behavior control system. The state variables of coalmine safety behavior control system consisted of both state variables of miner behavior system and manager behavior system. The output variable is safety or unsafe behavior of miner. The model implies that manager behavior control variables are the input coupling variables of miner behavior system, and the output variables of miner behavior system are input coupling variables of manager behavior system.

The output of safety behavior control system model is

$$
w(t)=g^{\prime \prime}\left(\boldsymbol{x}(t), \boldsymbol{y}(t), u_{5 t}, v_{1 t}, v_{2 t}, v_{3 t}, t\right)
$$

So the safety behavior control system model can be expressed as

$$
\min \sum_{t=0}^{T-1} w(t)=\sum_{t=0}^{T-1} g "\left(\boldsymbol{x}(t), \boldsymbol{y}(t), u_{5 t}, v_{1 t}, v_{2 t}, v_{3 t}, t\right)
$$

s.t $\quad \boldsymbol{x}(t+1)=\boldsymbol{f}(\boldsymbol{x}(t), \boldsymbol{u}(t), t) \quad x(0)$ is the known initial value

$$
\boldsymbol{y}(t+1)=\boldsymbol{h}(\boldsymbol{y}(t), \boldsymbol{v}(t), t) \quad y(0) \text { is the known initial value }
$$

As to the safety behavior control system, the manager's coal production task, manager's incentive contract, manager's cognition of work circumstance, and the work circumstance factors stimulating to miner, are all the extrinsic input variables that can influence the miner behavior. The manager's behavior control variables are the intrinsic variables that can influence the miner behavior. Therefore, although manager's behavior control variables are the important factors influencing miner behavior, the extrinsic variables play a more important role to miner behavior in the safety behavior control system. In order to better control the unsafe behavior of miners, government should strengthen the management of the extrinsic factors such as the coal production task, work circumstance, and manager's incentive contract.

\section{The Manager Optimal Control Behavior in Complete Information Condition}

The estimated value of unsafe behavior is the foundation basis for miner to make the choice of safe or unsafe behavior. In complete information condition, coal manager is aware of this estimated value of unsafe behavior. So the manager chooses his behavior according to this estimated value, not being influenced by his state factors. The manager's mission mainly is to depress miner's estimated value of unsafe behavior and encourage miner to adopt safe behavior using various management measures. This manager's mission can be decomposed into two aspects. On one aspect, coal manager needs to encourage miner to adopt safe behavior; on the other aspect, he also needs to cut down the total number of miner's unsafe behavior.

Because the miner makes the choice of safe or unsafe behavior based on the estimated value of unsafe behavior $w(t)$, the above management task can be described approximately by depressing the cumulated miner's estimated value of unsafe behavior in a period. Thus, coal mine safety problems faced by managers is to seek the optimal management behavior $\boldsymbol{u}(t)(t=0,1,2, \ldots, T)$ to minimize the cumulated miner's estimated value of unsafe behavior in a period. That is

$$
\min \sum_{t=0}^{T-1} w(t)=\sum_{t=0}^{T-1} g(\boldsymbol{x}(t), \boldsymbol{u}(t), t)
$$

s. $t \boldsymbol{x}(t+1)=\boldsymbol{f}(\boldsymbol{x}(t), \boldsymbol{u}(t), t) \times(0)$ is the known initial value. The Hamilton's function of this system is

$$
\begin{aligned}
& \boldsymbol{H}(\boldsymbol{x}(t), \boldsymbol{u}(t), \lambda(t+1), \mathrm{t}) \\
&= g(\boldsymbol{x}(t), \boldsymbol{u}(t), t)+\lambda(t+1) \boldsymbol{f}(\boldsymbol{x}(t), \boldsymbol{u}(t), t)
\end{aligned}
$$

The optimal necessity condition of this behavior control system is that taking various management measures to drive the Hamilton's function to its optimal value. When there is no constraint of management measures, it is

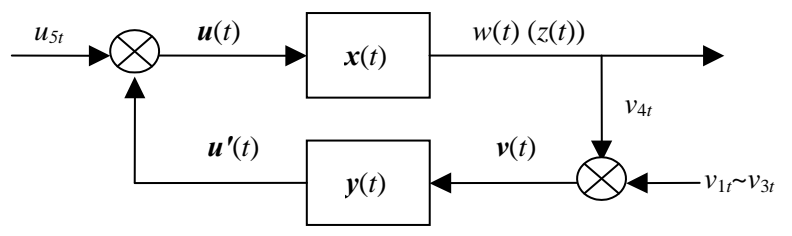

Figure 1. Coalmine safety behavior control system model 


$$
\begin{cases}\frac{\partial g}{\partial \boldsymbol{u}}+\lambda(t+1) \frac{\partial \boldsymbol{f}}{\partial \boldsymbol{u}}=0 & t=0,1,2, \ldots, T-1 \\ \lambda(t)=\frac{\partial g}{\partial \boldsymbol{x}}+\lambda(t+1) \frac{\partial \boldsymbol{f}}{\partial \boldsymbol{x}} & t=0,1,2, \ldots, T-1 \\ \boldsymbol{x}(t+1)=\boldsymbol{f}(\boldsymbol{x}(t), \boldsymbol{u}(t), t) & \boldsymbol{x}(0) \text { is the known initial value }\end{cases}
$$

This demonstrates that, with no resource constraint, manager's optimal management behavior consists of making full use of various management measures to let the marginal utility of various management measures on Hamilton's function equal to zero.

\section{The Manager Optimal Control Behavior in Incomplete Information Condition}

Different management measures have different effectiveness on the perception of miner's estimated value of unsafe behavior. It is foundational for manager to make his behavior choice on behalf of the coalmine. In complete information condition, the manager is easy to make his behavior choice as he aware of this estimated valuation. But in incomplete information condition, this estimated value is unknown to him, so the manager has a great space of decision-making.

Here, the management measures available to manager still consist of four factors, i.e. designing and providing production task and behavior criterion, carrying up rewards and punishment, bringing into effect of education and training, and creating group atmosphere and organizational climate. Suppose the miner's estimated value of unsafe behavior is $w(t)$, and the manager makes his behavior choice based on the estimated effects of various management measures. Thus, the manager's behavior variables will influence his management behavior, and these effects will eventually embody in miner's estimated value of unsafe behavior [17].

The following three kinds of behavior have an important influence on the behavior choice of coalmine manager:

1) Indifference: There are a lot of works to do for a manager, and controlling miners' unsafe behavior is only one of these works. It is very natural for a manager neglecting to control miners' unsafe behavior when he needs to deal with other seemingly more important works. This phenomenon is the manager indifference to control miner's unsafe behavior. The knowledge level and psychology state variables of a manager could influence this kind of choice behavior.

2) Incorrectness: Although manager thinks much of controlling miner's unsafe behavior, he maybe chooses the incorrect management measures. If so, this will inevitably unable to implement the management measures should be implemented. The knowledge level, psychol- ogy variables, and skill of manager influence this kind of behavior.

3) Passiveness: Manager maybe not works hard. This phenomenon is the manager irresponsible for his duties. For example, manager would be work inactively when his individual goals conflict with the coalmine objectives. The organizational loyalty and the expected reward from manager influence this kind of behavior.

On making choice of management measures, manager needs to estimate their different effects to miner's estimated value of unsafe behavior. This estimation is influenced by his inner factors, i.e. manager's mental state, knowledge state, physical state, organizational loyalty and work effort. All the influencing factors integrated may be described as manager's work initiative and work capability [18]. Let $w^{\prime}(t)$ denote miner's estimated value of unsafe behavior by manager, then it has

$$
w^{\prime}(t)=g^{\prime}(y(t), v(t), x(t), u(t), t)
$$

Similarly, the management task can be described as depressing the cumulated miner's estimated value of unsafe behavior estimated by the manager in a period. Thus, for coalmine manager, the task is to seek the optimal management behavior $\boldsymbol{u}(t)(t=0,1,2, \ldots, T)$ to minimize the cumulated miner's estimated value of unsafe behavior estimated by the manager in a period. So it has

$$
\min \sum_{t=0}^{T-1} w^{\prime}(t)=\sum_{t=0}^{T-1} g^{\prime}(\boldsymbol{y}(t), \boldsymbol{v}(t), \boldsymbol{x}(t), \boldsymbol{u}(t), t)
$$

s.t $\quad \boldsymbol{y}(t+1)=\boldsymbol{h}(\boldsymbol{y}(t), \boldsymbol{v}(t), t) y(0)$ is the known initial value. $\boldsymbol{x}(t+1)=\boldsymbol{f}(\boldsymbol{x}(t), \boldsymbol{u}(t), t) \times(0)$ is the known initial value.

The optimal necessity condition of this behavior control system can be obtained similarly. Its Hamilton's function is

$$
\begin{gathered}
\boldsymbol{H}\left(\boldsymbol{y}(t), \boldsymbol{v}(t), \boldsymbol{x}(t), \boldsymbol{u}(t), \lambda_{1}(t+1), \lambda_{2}(t+1), t\right) \\
=g^{\prime}(\boldsymbol{y}(t), \boldsymbol{v}(t), \boldsymbol{x}(t), \boldsymbol{u}(t), t)+\lambda_{1}(t+1) f(\boldsymbol{x}(t), \boldsymbol{u}(t), t) \\
+\lambda_{2}(t+1) \boldsymbol{h}(\boldsymbol{y}(t), \boldsymbol{v}(t), t)
\end{gathered}
$$

With no resource constraint, the optimal necessity condition of the behavior control system is

$$
\left\{\begin{array}{lc}
\frac{\partial g^{\prime}}{\partial \boldsymbol{u}}+\lambda_{1}(t+1) \frac{\partial \boldsymbol{f}}{\partial \boldsymbol{u}}=0 & t=0,1,2, \ldots, T-1 \\
\lambda_{1}(t)=\frac{\partial \boldsymbol{g}^{\prime}}{\partial \boldsymbol{x}}+\lambda_{1}(t+1) \frac{\partial \boldsymbol{f}}{\partial \boldsymbol{x}} & t=0,1,2, \ldots, T-1 \\
\boldsymbol{x}(t+1)=\boldsymbol{f}(\boldsymbol{x}(t), \boldsymbol{u}(t), t) & \boldsymbol{x}(0) \text { is the known initial value } \\
\frac{\partial \boldsymbol{g}^{\prime}}{\partial \boldsymbol{v}}+\lambda_{2}(t+1) \frac{\partial \boldsymbol{h}}{\partial \boldsymbol{v}}=0 & t=0,1,2, \ldots, T-1 \\
\lambda_{2}(t)=\frac{\partial g^{\prime}}{\partial \boldsymbol{y}}+\lambda_{2}(t+1) \frac{\partial \boldsymbol{h}}{\partial \boldsymbol{y}} & t=0,1,2, \ldots, T-1 \\
\boldsymbol{y}(t+1)=\boldsymbol{h}(\boldsymbol{y}(t), \boldsymbol{v}(t), t) & \boldsymbol{y}(0) \text { is the known initial value }
\end{array}\right.
$$


This optimal necessity condition maybe considered consists of two parts. The former three equations determine the optimal necessity condition of managing miner's behavior; the management objective is to minimize the cumulated miner's estimated value of unsafe behavior estimated by the manager in a period. The latter three equations determine the optimal necessity condition of managing manager own behavior; the management objective also is to minimize the cumulated miner's estimated value of unsafe behavior estimated by the manager in a period. The optimal necessity condition of the whole behavior control system can be achieved only when these two optimal conditions are met.

In order to facilitate a comparative analysis, the miner's estimated value of unsafe behavior by manager may be described as the form

$$
\boldsymbol{w}^{\prime}(t)=g^{\prime}(y(t), v(t), g(x(t), u(t), t), t)
$$

Then the optimal management measures of manager is decided by the optimal necessity condition of this behavior control system model

$$
\min \sum_{t=0}^{T-1} w^{\prime}(t)=\sum_{t=0}^{T-1} g^{\prime}(\boldsymbol{y}(t), \boldsymbol{v}(t), g(\boldsymbol{x}(t), \boldsymbol{u}(t), t), t)
$$

s.t $\boldsymbol{y}(t+1)=\boldsymbol{h}(\boldsymbol{y}(t), \boldsymbol{v}(t), t) y(0)$ is the known initial value. $\boldsymbol{x}(t+1)=\boldsymbol{f}(\boldsymbol{x}(t), \boldsymbol{u}(t), t) x(0)$ is the known initial value. Its Hamilton's function is

$$
\begin{aligned}
& \boldsymbol{H}\left(\boldsymbol{y}(t), \boldsymbol{v}(t), \boldsymbol{x}(t), \boldsymbol{u}(t), \lambda{ }_{1}(t+1), \lambda{ }_{2}(t+1), t\right) \\
& =g^{\prime}(\boldsymbol{y}(t), \boldsymbol{v}(t), g(\boldsymbol{x}(t), \boldsymbol{u}(t), t), t) \\
& +\lambda{ }_{1}(t+1) \boldsymbol{f}(\boldsymbol{x}(t), \boldsymbol{u}(t), t) \\
& +\lambda{ }_{2}(t+1) \boldsymbol{h}(\boldsymbol{y}(t), \boldsymbol{v}(t), t)
\end{aligned}
$$

The optimal necessity condition of the behavior control system is

$$
\left\{\begin{array}{lc}
\frac{\partial g^{\prime}}{\partial g} \frac{\partial g}{\partial \boldsymbol{u}}+\lambda_{1}^{\prime}(t+1) \frac{\partial \boldsymbol{f}}{\partial \boldsymbol{u}}=0 & t=0,1,2, \ldots, T-1 \\
\lambda_{1}^{\prime}(t)=\frac{\partial \boldsymbol{g}^{\prime}}{\partial g} \frac{\partial g}{\partial \boldsymbol{x}}+\lambda_{1}^{\prime}(t+1) \frac{\partial \boldsymbol{f}}{\partial \boldsymbol{x}} & t=0,1,2, \ldots, T-1 \\
\boldsymbol{x}(t+1)=\boldsymbol{f}(\boldsymbol{x}(t), \boldsymbol{u}(t), t) & \boldsymbol{x}(0) \text { is the known initial value } \\
\frac{\partial g^{\prime}}{\partial \boldsymbol{v}}+\lambda_{2}^{\prime}(t+1) \frac{\partial \boldsymbol{h}}{\partial \boldsymbol{v}}=0 & t=0,1,2, \ldots, T-1 \\
\lambda_{2}^{\prime}(t)=\frac{\partial g}{\partial \boldsymbol{y}}+\lambda_{2}^{\prime}(t+1) \frac{\partial \boldsymbol{h}}{\partial \boldsymbol{y}} & t=0,1,2, \ldots, T-1 \\
\boldsymbol{y}(t+1)=\boldsymbol{h}(\boldsymbol{y}(t), \boldsymbol{v}(t), t) & \boldsymbol{y}(0) \text { is the known initial value }
\end{array}\right.
$$

Here $\frac{\partial g^{\prime}}{\partial g}$ describers the relationship between miner's estimated value of unsafe behavior by the manager and by the miner. It gives us the information of what is the effectiveness of various management measures on miner's estimated value of unsafe behavior. The former three equations determine the optimal necessity condition of managing miner's behavior, and the latter three equations determine the optimal necessity condition of managing manager own behavior. The optimal necessity condition of the whole behavior control system is achieved only when these two optimal conditions are met in the same time.

With regard to the effectiveness of various management measures on miner's estimated value of unsafe behavior, there is a theorem.

Theorem: If $\frac{\partial g^{\prime}}{\partial g}=1$, the optimal management behavior in safety behavior control system of (15) is the same as that of (9).

The proof of this theorem is omitted here.

The theorem implies that, when manager's behavior factors influence his understanding of miner's estimated value of unsafe behavior, his choice of management behavior definitely is not the optimal one. Because it is difficult to take the optimal management behavior for the manager, it is inevitable there are managerial defects even errors in the coalmine safety management.

\section{Discussion and Conclusions}

We propose a model of safety behavior control system to discuss some issues of coalmine manager behavior, and analyze the conditions of optimal management behavior of coalmine manager both in complete information and incomplete Information condition. The following conclusions could be arrived.

1) The management measures of coalmine manager are the important factors influencing miner behavior. However, manager's production tasks, manager's incentive contract, manager's cognition of work circumstance take more important roles on the point of whole safety behavior control system. In order to better control the unsafe behavior of miners, government should strengthen the management of the extrinsic factors such as the coal production task, work circumstance, and manager's incentive contract.

2) The manager's inner factors do not influence his management behavior in complete information condition, but they influence it in incomplete information condition. The necessity condition of optimal manager behavior choice consists of two blocks, one block determines the optimal necessity condition of managing miner's behavior, and the other block determines the optimal necessity condition of managing manager own behavior. The optimal necessity condition of the whole behavior control system is achieved only when these two blocks' optimal 
conditions are met in the same time.

3) Only when the miner's estimated value of unsafe behavior by manager is same as the miner's estimated value by himself, the manager's optimal behavior choice is possible. Because the manager's behavior factors influence his understanding of miner's estimated value of unsafe behavior unavoidably, it is difficult for the manager to obtain the optimal management behavior in the real world. It could well explain why it is inevitable there are managerial defects even errors in the coalmine safety management.

As discussed above, in coalmine safety behavior control system, manager is the subject of implementation of management measures, miner is the object of implementation of management measures, both of them have the ability to learn and adapt, and would make choice by themselves judgments.

In the coal production, the purpose of manager and miner is to achieve their respective behavior value. For a miner, this kind of behavior value is achieved by his specific production activities, so what determines the miner's behavior is his perceived value of various production activities. But for a manager, this kind of behavior value is achieved by his management activities, so what determines the manager's behavior is his perceived value of various management activities.

In order to make coal production safe enough, the manager first needs to determine the safety level of coal production based on the coal production task and the actual production condition. Then, according to this safety level, he needs to make the choice of various available management measures. In this way, what determines the manager's behavior choice is the effectiveness of different management measures to the miner's unsafe behavior.

Furthermore, the manager's behavior choice is based on his understanding of miner's estimated value of unsafe behavior, and this cannot but influenced by his own behavior variables, especial influenced by his own specific perception and judgments in the work. So in order to make a proper behavior choice, manager should try to avoid influencing by own state variables. Therefore, he needs to strengthen self-behavior management.

How to strengthen self-behavior management for a manager, and how to inspirit the manager to strengthen self-behavior management, are the problems needed to be studied carefully in the near future.

\section{Acknowledgement}

This research is supported by the National Natural Science Foundation of China under Grant 70871113 and the Humanities and Social Science Research Foundation of Ministry of Education of the People's Republic of China (Project ID: 07JA630025).

\section{REFERENCES}

[1] Z. Y. Lin and L. C. Xu, "Analysis of the human error and its reasons in coal mine accidents," Chinese Journal of Ergonomics, Vol. 2, No. 2, pp. 17-20, 1996.

[2] Q. R. Cao and X. F. Song, "Problems being faced with the unsafe behavior study and its countermeasure," China Coal, Vol. 32, No. 11, pp. 62-63, 2006.

[3] H. Mintzberg, "The nature of managerial work," Harper \& Row, New York, 1973.

[4] P. Allan, "Managers at work: A large-scale study of the managerial job in the New York city government," Academy of Management Journal, Vol. 24, No. 3, pp. 613619, 1981.

[5] G. Yukl, S. Wall, and R. Lepsinger, "Preliminary report on validations of the managerial practices survey," In Measures of leadership, K. E. Clark and M. B. Clark, Eds., West Orange, NJ, 1989.

[6] D. Schaechtel, "How to build a safety management system?” Professional Safety, Vol. 42, No. 8, pp. 22-24, 1997.

[7] R. Jens, "Risk management in a dynamic society: A modeling problem," Safety Science, Vol. 27, No. 2-3, pp. 183-213, 1997

[8] S. R. Sun and L. P. Qi, "Developments of mathematical models of behavior control mechanism and behavior management system,” Journal of University of Shanghai for Science and Technology, Vol. 26, No. 1, pp. 47-52, 2004.

[9] P. Jason, D. Pasquale, and E. S. Geller, "Critical success factors for behavior-based safety: A study of twenty industry-wide applications," Journal of Safety Research, Vol. 30, No. 4, pp. 237-249, 1999.

[10] O. Michael, “The relationship between employees' perceptions of safety and organizational culture,” Journal of Safety Research, Vol. 33, No. 1, pp. 231-243, 2002.

[11] E. S. Geller, "Working safe: How to help people actively care for health and safety," Second Edition, Lewis Publishers, Inc., 2001.

[12] M. Neal, A. Griffin, and P. M. Hart, "The impact of organizational climate on safety climate and individual behavior,” Safety Science, Vol. 34, pp. 99-109, January 2000.

[13] M. Jane, "Investigating factors that influence individual safety behavior at work," Journal of Safety Research, Vol. 35, pp. 275-285, January 2004.

[14] Q. R. Cao, "Analysis of the influencing factors of coal miner unsafe behavior,” Mining Safety \& Environmental Protection, Vol. 33, No. 6, pp. 80-82, 2006.

[15] Q. R. Cao, “A Study on the unsafe behavior in the coal mining and its control system,” Ph. D. dissertation, Department of Science and Technology, China University of Mining and Technology, Xuzhou, 2005.

[16] Q. R. Cao and X. F. Song, "Study on the unsafe behavior of coal miner and its management avenue,” Economic Management, Vol. 15, pp. 62-65, July 2006. 
[17] M. Grabowski and H. R. Karlene, "Human and organizational error in large scale systems," IEEE Transaction on System, Man, and Cybernetics-part A: System and Human, Vol. 26, No. 1, pp. 2-16, 1996.
[18] Q. R. Cao, "Study on the cognitive diversities between managers and workers in controlling unsafe behavior," China Safety Science Journal, Vol. 17, No. 1, pp. 22-28, 2007. 